\title{
REVISIÓN HISTÓRICA SOBRE LA EPIDEMIOLOGÍA MATEMÁTICA
}

\author{
HISTORICAL REVIEW ON MATHEMATICAL \\ EPIDEMIOLOGY
}

Roberto Ávila Pozos ${ }^{1}$ Daniel Zuñiga Rangel ${ }^{2}$

\section{Resumen}

En la actualidad el estudio de las enfermedades infecciosas es un tema de interés para la población. Especialmente para los especialistas que se dedican a la planificación de programas de control; es por esto que deben apoyarse de otros métodos, los cuales brinden mayor información acerca de los procesos infecciosos. La aplicación de las matemáticas en la epidemiología ha tenido un gran auge debido a que la modelación permite estudiar qué factores intervienen en el desarrollo de un brote epidemiológico. Estos estudios se remontan hasta 1760 cuando Daniel Bernoulli propuso un modelo matemático acerca de la peste. Más tarde Kermack y Mckendrick del Laboratory of the Royal College of Physicians en Edinburgo, formularon un modelo matemático para describir la epidemia de peste que sufrió la India en 1906. Este modelo fue considerado durante el siglo XX uno de los pilares que sustentó el desarrollo de la epidemiología matemática. La epidemiología matemática proporciona herramientas útiles, las cuales ayudan a determinar y diseñar programas en general, de políticas de salud pública.

Palabras clave: Enfermedades infecciosas, epidemiología, epidemiología matemática.

\begin{abstract}
At present the study of infectious diseases is an issue of interest to the population. Especially for specialists engaged in planning control programs. Therefore specialists should use other methods, which provide more information about infectious processes. The application of mathematics in epidemiology has had a boom because the modeling allows to study the factors involved in the development of an epidemic outbreak. These studies date back to 1760 when Daniel Bernoulli proposed a mathematical model about the plague. Later Kermack and Mckendrick of the Laboratory of the Royal College of Physicians in Edinburgo, they formulated a mathematical model to describe the plague that India suffered in 1906. This model was considered during the twentieth century one of the pillars that supported the development of mathematical epidemiology. The mathematical epidemiology provides useful tools, which help determine and design planning programs in general, public health policies.
\end{abstract}

Keywords: infectious diseases, epidemiology, mathematical epidemiology. 


\section{INTRODUCCIÓN}

A lo largo de la historia, la humanidad se ha enfrentado a un gran número de brotes epidémicos; estos han provocado un severo impacto en la densidad de la población humana. Por mencionar algún ejemplo, la caída del imperio Azteca en manos de Cortés, se debió en gran parte a una epidemia de viruela que azotó a la población mesoamericana. Entre los años 1519-1530 la población mexicana pasó de 30 millones de habitantes a solo 3 millones (Brauer y Castillo, 2012).

Sin embargo en la actualidad, a pesar de los distintos avances en tratamientos médicos y en el desarrollo de nuevos fármacos, no hemos estado exentos de brotes epidémicos. Es por eso que el estudio de las enfermedades infecciosas es un tema de gran importancia para la sociedad. En estos años hemos sido testigos de los efectos de muchas enfermedades tales como el VIH, el dengue, la influenza A(H1N1), etc. Tan sólo en 2014 fallecieron cerca de 1.2 millones de personas en el mundo y a mediados del 2015 se registraron 15.8 millones de personas infectadas por el VIH (OMS, 2015). Otra enfermedad vírica es el dengue; este virus se transmite por mosquitos hembra, por las especies Aedes aegypti y Aedes albopictus. Estos mosquitos también son transmisores del virus de Chikungunya y la infección del virus del Zika. Se estima que entre 50 y 100 millones de personas se infectan anualmente (Buczack et al., 2012).

Las políticas de salud pública están orientadas a la prevención de futuros brotes epidémicos; una de ellas son las campañas de vacunación. Las vacunas tienen como principal objetivo proporcionar inmunidad, previniendo alguna enfermedad. Una campaña de vacunación tendrá como propósito reducir el número de infecciosos, así como brindar inmunidad a sujetos susceptibles. Sin embargo, en muchos otros casos no existen vacunas, como es el caso del SIDA y de la tuberculosis. Es por eso que los especialistas en epidemias deben apoyarse de otros métodos, los cuales brinden mayor información acerca de los procesos infecciosos. El estudio de los procesos infecciosos mediante modelos matemáticos constituye una herramienta muy útil para establecer predicciones y explicaciones del por qué el número de casos nuevos fluctúa de cierta manera. Además los modelos matemáticos sirven para interpretar tendencias epidemiológicas que contribuyen a la organización y la recolección de datos, y así diseñar programas de control de enfermedades.

\section{ANTECEDENTES DE LA EPIDEMIOLOGÍA MATEMÁTICA}

La aplicación de las matemáticas a la epidemiología puede remontarse hasta 1760 cuando Daniel Bernoulli un matemático de los Países Bajos publicó un tratado sobre la epidemia de la peste (Velasco, 2007), la cual asoló a Europa durante los años 1665-1666.

En 1906 Hamer propuso la ley de acción de masas, en donde, el número de contactos que produce una enfermedad por unidad de tiempo es proporcional al número total de contactos entre individuos infecciosos y sanos.

Ronald Ross un médico escocés, él comenzó sus estudios acerca de la malaria en 1892. Dos años después decidió investigar de manera experimental que los mosquitos estaban relacionados con 
la propagación de dicha enfermedad en India. Ross estaba interesado por la creación de medidas de prevención contra la malaria, realizó encuestas en lugares como África Occidental, Grecia y Chipre.

Ganó el premio nobel en medicina en el año de 1902 debido a su descubrimientos de las causas que provocan la malaria. Después Ronald Ross formuló un modelo matemático en su libro "The prevention of Malaria" en 1911 (Velasco, 2007), el cual utilizó con el propósito de erradicar dicha enfermedad, ya que no era necesario extinguir a los mosquitos, sino disminuir su población. Esta idea fue rechazada en aquellos tiempos, ya que se creía que la cantidad de enfermos no estaba relacionada con la densidad de la población de mosquitos.

Ross desarrolló un método que consistía en suponer hipótesis de las posibles causas de un brote epidémico. Posteriormente formulaba un sistema de ecuaciones diferenciales para describir el brote; después analizaba sus resultados que obtenía con sus ecuaciones y las implicaciones epidémicas. Finalmente comparaba sus predicciones con los datos previamente capturados.

Más tarde en 1927 W.O. Kermack y A.G. McKendrick del Laboratory of the Royal College of Physicians en Edinburgo, dieron seguimiento a las ideas de Ross, formulando un modelo matemático para describir la epidemia de peste que sufrió la India en 1906 (Velasco, 2000). Es un modelo en el cual se hacen algunos supuestos por ejemplo en las tasas de flujo entre las diferentes clases en las que está dividida la población.

\section{MODELO DE KERMACK Y MCKENDRICK}

En los modelos matemáticos la población se divide en tres clases: susceptible, infeccioso y recuperado. Las personas pueden pasar de una clase a otra. Los modelos matemáticos tratan de mostrar este flujo mediante ecuaciones diferenciales entre las distintas clases en las que está dividida la población. Este tipo de modelos son llamados de compartimentos. Además los modelos matemáticos no tratan de mostrar la cantidad de casos fatales que pueden ocurrir, más bien estudian los factores que contribuyen a la erradicación de una epidemia.

El modelo de Kermack y McKendrick fue durante el siglo XX uno de los pilares que sustentó el desarrollo de la epidemiología matemática.

Para la formulación del modelo de Kermack y McKendrick se divide a la población en tres clases excluyentes que serán designadas por las letras $S(t), I(t)$ y $R(t)$, donde $S(t)$ denotará el número de individuos susceptibles de contraer la enfermedad en un determinado tiempo $t ; I(t)$ el número de individuos capaces de transmitir la enfermedad, es decir el número de infecciosos al tiempo $t ; R(t)$ el número de individuos que han perdido la posibilidad de ser infectados; ya sea por haber sido aislados del resto, por haber sido inmunizados o por haber fallecido a consecuencia de la enfermedad. Denotamos a $N$ como la población total al tiempo $t$, donde $N=$ $S(t)+I(t)+R(t)$.

El modelo es el siguiente: 


$$
\begin{gathered}
\frac{d S(t)}{d t}=-\beta S(t) I(t) \\
\frac{d I(t)}{d t}=\beta S(t) I(t)-\frac{1}{\tau} I(t) \\
\frac{d R(t)}{d t}=\frac{1}{\tau} I(t)
\end{gathered}
$$

Donde $\beta$ representa la tasa de transmisión de la enfermedad y $\tau$ la tasa de recuperación o muerte. Kermack y McKendrick establecieron los siguientes postulados:

a) La enfermedad tenía que ser viral o bacteriana y que pudiera ser transmitida por contacto directo entre personas.

b) Al comienzo de la epidemia únicamente una fracción de la población es infecciosa.

c) La población se supone constante. Todas los individuos son susceptibles de enfermarse, salvo por las personas que inicialmente son enfermas.

d) Todos los individuos sufren el curso completo de la enfermedad. Al final se recuperan adquiriendo inmunidad, o mueren.

\section{DISEÑO DE PROGRAMAS DE VACUNACIÓN}

Lo anterior hacía referencia a una parte del desarrollo de la epidemiología matemática, pero existen muchas dudas que el lector podrá notar respecto al uso real de los modelos matemáticos, ya que hasta este momento no hemos explicado de manera concisa como se utilizan realmente este tipo de modelos, ni cómo influyen en el diseño de programas de vacunación.

Actualmente la inmunización es el principal método para la prevención de enfermedades. El comportamiento de una persona respecto a un agente infeccioso es un proceso muy complejo, pues depende de diversos factores como el curso de la infección en el sujeto, la biología del agente infeccioso, tratamiento médico que esté llevando, historial clínico, etc. Es por esto que predecir los efectos de una campaña de vacunación es sumamente complicado, también es casi imposible modelar toda la complejidad que implica un proceso biológico.

Para la construcción de un modelo es importante tener información cuantitativa de períodos de latencia, infecciosos e incubación. El período de latencia se define como el período desde el momento en que un individuo se infecta hasta el punto en que se vuelve infeccioso. El período infeccioso es el período en el que un individuo es capaz de transmitir la enfermedad. El período de incubación es el período una vez que se inicia la infección hasta que se presentan síntomas. 
Del modelo anterior, Kermack y Mckendrick derivaron un teorema que demuestra la existencia de una constante conocida como "número reproductivo básico" representado por $R_{0}$. Se considera como el número promedio de casos nuevos que genera un individuo infeccioso en una población susceptible durante el tiempo que permanece infeccioso. Su magnitud determina si una enfermedad será abatida (cuando $R_{0}<1$ ); es decir el número de individuos infecciosos decrecerá hasta llegar a cero; o si se produce un brote epidémico, en este caso el número de individuos infecciosos crece (cuando $R_{0}>1$ ), alcanzando un máximo, y posteriormente decrece a cero. Las campañas de vacunación logran disminuir el número reproductivo básico, evitando que rebase el valor umbral.

¿Pero de qué manera se construye un programa de vacunación eficiente utilizando estas ideas acerca del $R_{0}$ ? En 1993 Karl Hadeler de la Universidad de Tubingen encontró que el número reproductivo de vacunación se podía escribir como una resta del reproductivo básico y otro que incorpora el efecto de vacunación (Velasco, 2007). Este segundo término está en función de la diferencias en las tasas de infección entre los individuos vacunados y no vacunados. Entonces el objetivo de una buena estrategia de vacunación consiste en aumentar el segundo término, para que decrezca el número reproductivo de vacunación.

Hadeler identificó 2 casos óptimos; el primero es cuando se vacuna únicamente a los individuos susceptibles y el segundo, cuando se vacuna a cualquier individuo sin considerar su estado inmunológico. Para ambos caso las mejores estrategias fueron:

- Vacunar a toda la población en una determinada edad.

- Vacunar a una parte de la población en alguna edad A y el resto a una edad B.

Pero ¿se han utilizado este tipo de ideas para la planificación de programas de vacunación? La respuesta es sí. Algunos de los ejemplos más claros son los modelos de Longini, Ackerman y Elveback mediante el cual decidieron que grupos de ciertas edades debían vacunarse para minimizar el riesgo de muertes en una epidemia de influenza en 1978. Más tarde, los modelos de Hethcote y Yorke en el año de 1984, compararon los procedimientos para el control de la gonorrea en la población de Estados Unidos (Miramontes et al., 2002).

\section{CONCLUSIONES}

La aplicación de las matemáticas a la epidemiología ha sido de gran relevancia, ya que los modelos matemáticos constituyen una herramienta que sirve para establecer predicciones, estructurar información de los procesos biológicos, e influyen en el diseño de planificación de programas de vacunación.

El lenguaje matemático proporciona que las predicciones que establece estén libres de ambigüedades, sin embargo es importante considerar que los modelos están sustentados en ciertas hipótesis, lo que se traduce en ciertas limitaciones, y es claro para el lector que modelar todos los procesos que implica el desarrollo de un brote epidémico es sumamente complicado. Sin embargo el uso de modelos más sofisticados permitirá ayudar a interpretar de manera más clara 
las tendencias epidemiológicas y así diseñar mejores programas para el control de las enfermedades.

\section{REFERENCIAS}

Brauer, F., Castillo-Chávez, C. (2001)Mathematical Models in Population.Biology and Epidemiology. Second edition. Springer, 522 pp.

Brauer, F., Castillo-Chávez, C., De La Pava, E., Barley, K., Castillo-Garsow, C.W., Chowell, D., Espinoza, B., Gonzalez-Parra, P., Hernandez-Suarez, C., Moreno, V. M. (2014) Modelos de la propagación de enfermedades infecciosas. Primera edición. Programa Editorial Dirección de Investigaciones y Desarrollo Tecnológico, 176 pp.

Nokes, D.J., Anderson, R. M. (1988) The use of mathematical models in the epidemiological study of infectious diseases and in the design of mass immunization programmes. Epidemiology and infection. 101(1), 1-20.

Buczak,Anna L.,Koshute,P.T.,Babin,S.M.,Feighner,B.H.,Lewis ,S.H. (2012) A data-driven epidemiological prediction method for dengue outbreaks using local and remote sensing data. BMC Medical Informatics and Decision Making. 12, 1-20.

Miramontes,P., Sánchez-Garduño,F.,Gutiérrez,J.L. (2002) Clásicos de la Biología Matemática, Primera edición 2002. Siglo XXI, 186 pp.

Velasco-Hernández,J.X. (2007) Modelos Matemáticos en epidemiología: enfoques y alcances. Miscelánea Matemática. 44,11-27.

Velasco-Hernández,J.X. (2000) El gen, la forma, el virus y la idea: una perspectiva personal de la biología matemática. Miscelánea Matemática. 32, 5-38

Velasco-Hernández, J. X. (1999) Sobre enfermedades infecciosas. Miscelánea Matemática. 29, $51-72$.

\footnotetext{
${ }^{1}$ Profesor investigador en el Área Académica de Matemáticas y Física de la Universidad Autónoma del Estado de Hidalgo.Doctor en Ciencias por Universidad Autónoma Metropolitana. Sus temas de investigación son biomatemáticas

${ }^{2}$ Daniel Zuñiga Rangel. Estudiante de la Licenciatura en Matemáticas Aplicadas, Universidad Autónoma del Estado de Hidalgo, danizrangel@gmail.com
} 